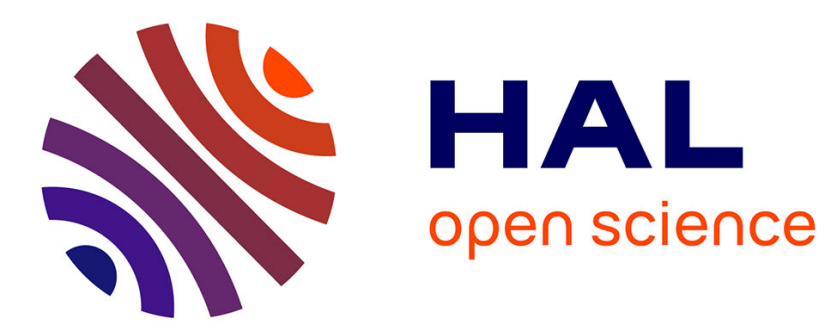

\title{
De jeunes parents dans la tourmente. Stratégies de recours et gestion des normes de parentalité à l'arrivée du premier enfant.
}

\author{
Marion Manier
}

\section{- To cite this version:}

Marion Manier. De jeunes parents dans la tourmente. Stratégies de recours et gestion des normes de parentalité à l'arrivée du premier enfant.. Revue des politiques sociales et familiales, 2018, 127. halshs-02139278

\author{
HAL Id: halshs-02139278 \\ https://shs.hal.science/halshs-02139278
}

Submitted on 24 May 2019

HAL is a multi-disciplinary open access archive for the deposit and dissemination of scientific research documents, whether they are published or not. The documents may come from teaching and research institutions in France or abroad, or from public or private research centers.
L'archive ouverte pluridisciplinaire HAL, est destinée au dépôt et à la diffusion de documents scientifiques de niveau recherche, publiés ou non, émanant des établissements d'enseignement et de recherche français ou étrangers, des laboratoires publics ou privés. 


\section{De jeunes parents dans la tourmente Stratégies de recours et gestion des normes de parentalité à l'arrivée du premier enfant}

Mots-clés

- Normes

- Parentalité

- Premier enfant

- Recours/non-recours

Marion Manier Chargée de recherche à la caisse d'Allocations familiales des Alpes-Maritimes.

Chercheuse associée à I'Université Nice Sophia Antipolis - Urmis

(CNRS UMR 8245/IRD UMR 205).

De nombreux travaux consacrés tant aux évolutions des formes familiales qu'aux politiques familiales qui les visent ont montré que l'émergence de la notion de " parentalité » semble tout à la fois produire et traduire un renouvellement des normes familiales et parentales. La notion s'inscrit dans la reconnaissance progressive de la diversification des organisations familiales, mais elle devient aussi I'objet $d^{\prime}$ une régulation politique de la famille de plus en plus affirmée autour du " centrage sur l'enfant " (Bachmann et al., 2016, p. 21). S'appuyant sur le registre de l'intérêt supérieur de l'enfant, les normes sociales ${ }^{(1)}$ régissant la parentalité, portées par les politiques sociales, familiales et éducatives, s'accompagnent ainsi de fortes attentes sociales à l'égard des familles. On assiste à ce que nombre d'auteurs qualifient de processus de « responsabilisation » - voire de " surresponsabilisation » - des parents, en particulier auprès des familles les plus pauvres (Martin, 2003 ; Boucher, 2011) et à l'égard des mères, plus spécifiquement ciblées par les dispositifs et les interventions (Bachmann et al., 2016 ; Chauvière, 2008 ; Vozari, 2011, Blöss, 2016). L'injonction à être de "bons parents " (Martin, 2014), à " réussir » en tant que parents [Singly (de), 2009] se décline en effet de manière variable selon les "vecteurs de production de normes " (Chauffaut, Dauphin, 2012, p.109) qui la relaient (en particulier les institutions dans le domaine de la santé, la petite enfance, la parentalité, la famille) et selon les parents auxquels elle $\mathrm{s}^{\prime} \mathrm{adresse}^{(2)}$.

Les messages et les normes diffusés par les professionnels que les jeunes parents sont amenés à rencontrer - dans le champ social, éducatif ou sanitaire - sont d'abord fortement marqués par les rapports sociaux de sexe, instituant des modèles de maternité et de paternité très différenciés (Bachmann et al., 2016 ; Boyer et Céroux, 2010), et ce dès la grossesse (Menuel, 2012). Ils sont également marqués par des différenciations socioéconomiques et culturelles, instituant des distinctions entre des pratiques savantes - valorisées parmi les classes moyennes ou supérieures et des pratiques " traditionnelles ", " populaires ", voire «naturelles» (Gojard, 2000 et 2010 ; Geay, 2014). Si les mécanismes de production et de diffusion des conceptions normatives de la parentalité ont fait l'objet d'un certain nombre d'analyses et semblent relativement bien connus, qu'en est-il des effets de ces prescriptions sur les familles, de leur réception par les parents?

L'un des objectifs de cet article est de considérer ces enjeux normatifs du point de vue des parents, à un moment où ils s'y confrontent pour la première fois, à l'arrivée de leur premier enfant. À partir des résultats d'une enquête sociologique qualitative menée en 2016, sont ainsi explorées les pratiques et les stratégies mises en place par les parents face aux avis, conseils, prescriptions ou injonctions normatives qui leur sont adressés, à travers l'analyse de leurs rapports aux différents interlocuteurs auxquels ils ont affaire ou auxquels ils ont recours (professionnels de santé, de la petite enfance, famille et entourage) : quelles normes de parentalité leur sont adressées par ces différents interlocuteurs, comment se déclinent-elles selon les milieux sociaux et le genre des parents?

Quelles relations les jeunes parents tissent-ils avec ces différents interlocuteurs et comment reçoivent-ils leurs avis, leurs conseils ou leurs prescriptions ? Enfin, quelles stratégies de recours mais aussi de mise à distance, de sélection, d'évitement ou de résistance à ces interlocuteurs mettent-ils en place?

Comme le montre une enquête réalisée par la Caisse nationale des Allocations familiales (Cnaf) sur les attentes

\footnotetext{
(1) Par «normes sociales », on entend « des règles qui orientent les conduites sociales, les manières d'agir et de se comporter, en définissant ce qui est désirable, acceptable ou moral et ce qui ne l'est pas dans un groupe social donné. Ces règles se manifestent sous la forme de modèles et de sanctions positives ou négatives à s'y conformer. »

(2) Les recherches sur les questions de la parentalité soulignent bien souvent les ambivalences d'un certain nombre de politiques ou de dispositifs pris entre des logiques d'accompagnement à la parentalité pour tous et des logiques plus ciblées, de « prévention » voire de contrôle auprès des catégories populaires. Voir notamment au sujet de ces ambivalences Martin (2003), Vozari (2011), Chauvière (2008), Neyrand (2011), Pioli (2006).
}

\begin{tabular}{l|l} 
Revue des politiques sociales et familiales & $n^{\circ} 127-2^{e}$ trimestre 2018
\end{tabular}

49 Parentalité 
et les besoins des parents (Moeneclaey et Crépin, 2016), $43 \%$ des parents perçoivent I'exercice de leur rôle de parents comme difficile. L'arrivée d'un enfant fait partie des périodes qu'ils identifient comme déstabilisantes. Faisant naître, au moins provisoirement, une multitude de questionnements et d'incertitudes, la venue d'un enfant

Une enquête qualitative auprès de parents sur l'arrivée du premier enfant

\section{L'enquête}

Cet article s'appuie sur les résultats d'une enquête sociologique menée dans le cadre d'une collaboration de recherche entre la caisse d'Allocations familiales des Alpes-Maritimes et I'Université Nice Sophia Antipolis (Unité de recherche Urmis) en 2016 intitulée " Jeunes parents dans la tourmente? Vécu, attentes et recours des parents à l'arrivée du premier enfant ». L'objectif de cette enquête était à la fois d'explorer l'expérience et le vécu des parents à l'arrivée du premier enfant et d'interroger leurs pratiques et leurs attentes en matière d'information, de ressources et de recours à des professionnels (santé, enfance, parentalité).

\section{Les parents enquêtés}

Trente et un entretiens semi-directifs approfondis ont été réalisés auprès de futurs parents (au cours de la grossesse) ou de parents de jeunes enfants (de moins de 3 ans) afin de saisir différents moments de l'expérience de parents : vingt-quatre mères et sept pères - dont cinq célibataires et vingt-six en couple - , parents d'enfants âgés de moins de 3 ans (dont vingt parents d'un seul enfant), résidant dans les Alpes-Maritimes, vingt et un en zone urbaine et dix en zone rurale ou de montagne).

Les parents ont été rencontrés par l'intermédiaire de professionnels de la petite enfance et de travailleurs sociaux, ce qui a occasionné certains biais de sélection (une surreprésentation des femmes et de parents diplômés). Toutefois, les situations socioprofessionnelles des parents rencontrés sont variées (demandeurs d'emploi, étudiants, employés, professions intermédiaires, cadres et cadres supérieurs sont représentés) ainsi que les niveaux de formation (de la classe de cinquième au collège à un niveau doctorat).

\section{La méthodologie}

La méthodologie adoptée s'appuie sur une démarche qualitative par entretiens. La finalité étant de recueillir l'expérience et le sens donné au fait de devenir parent à l'arrivée d'un premier enfant (depuis la grossesse jusqu'aux 3 ans de l'enfant), les entretiens réalisés ont duré en moyenne une heure trente à deux heures. Ils ont permis de recueillir des récits détaillés et sensibles de I'arrivée d'un premier enfant. Par ailleurs, parmi cet échantillon de trente et une personnes, quatre couples ont été interviewés (dont deux auprès des deux parents réunis et deux auprès des deux parents séparément). Ce dispositif a permis de mettre en perspective les points de vue des mères et des pères sur leurs préoccupations et leurs rôles parentaux respectifs. implique aussi une réorganisation du quotidien, du couple, de l'activité professionnelle et domestique, en particulier pour les femmes (Régnier-Loilier, 2009 ; Pailhé et Solaz, 2006). C'est à ce double titre que les jeunes parents s'engagent bien souvent dans une quête de réponses, d'informations, de soutien ou de réconfort. Ces réajustements se combinent également à l'entrée dans une période de socialisation à de nouveaux rôles, de nouveaux codes et de nouvelles normes mais aussi de nouveaux interlocuteurs. Véritable "rite d'institution » pour reprendre la formule de Nathalie Oria et Jérôme Camus (2012), l'arrivée d'un premier enfant soumet les jeunes parents à I'encadrement et à l'intervention de différentes institutions et de différents professionnels. Dès lors, comme le montre Séverine Gojard (2010), I'arrivée d'un enfant, en confrontant les parents à une série de questionnements, les expose aussi à une pluralité de "prescripteurs » de normes : les jeunes parents font face, en effet, à des interlocuteurs détenteurs d'une expertise, d'une légitimité voire d'une certaine autorité symbolique, dont la parole peut se révéler $\mathrm{d}^{\prime}$ autant plus " prescriptive »-c'est-à-dire se manifestant sous la forme de recommandations relativement formelles et contraignantes - qu'eux-mêmes sont encore peu sûrs d'eux. Les professionnels de la santé, de la petite enfance ou de la parentalité, tout comme l'entourage et la famille, sont ainsi de potentiels recours mais aussi d'éventuels prescripteurs de normes pour les parents, à plus forte raison à l'arrivée d'un premier enfant.

\section{Les interlocuteurs des jeunes parents et leurs normes}

Les interlocuteurs des jeunes parents apparaissent comme des acteurs majeurs, des figures incontournables de l'expérience parentale, et les messages qu'ils adressent aux jeunes parents revêtent un poids important.

\section{Les professionnels de santé et de la petite enfance : au-delà des normes de la profession}

Les professionnels de la santé - médecins généralistes, gynécologues, sages-femmes, puéricultrices, auxiliaires de puériculture, pédiatres... - sont des interlocuteurs inévitables des jeunes parents. Incarnant aux yeux de la plupart d'entre eux des références en matière d'expertise, ils sont un recours privilégié des parents en recherche d'informations fiables et scientifiques concernant la santé de la mère et de l'enfant. Les parents disent notamment compter sur ces professionnels pour les aider « à faire le tri » parmi la multitude d'informations et d'avis différents qu'ils rencontrent dans leur entourage, sur Internet, dans 
la documentation. Or, au-delà des aspects strictement médicaux et sanitaires, les professionnels de santé sont à la fois sollicités et à l'initiative de conseils sur des dimensions beaucoup plus sociales de la grossesse, la naissance, I'allaitement, la puériculture, l'alimentation, les comportements maternels et infantiles. La parole médicale participe ainsi, de manière plus ou moins explicite et plus ou moins intentionnelle, à relayer des normes qui sont aussi sociales et morales; des normes parfois perçues par les parents comme des critères de définition et d'évaluation d'une « bonne parentalité ».

- Virginie, demandeuse d'emploi, en couple, 36 ans, raconte ainsi les remarques de certains professionnels de santé qu'elle a reçues comme une mise en question de sa capacité à être une " bonne mère » : "La sagefemme à domicile est venue, et alors là, pour me déstabiliser, il n'y avait rien de mieux ! Elle a pris la température de Léo, bon c'était l'hiver, il était habillé tout à fait normalement et il a 36 et quelques, et là : "Mais votre enfant il a froid! Faut I'habiller plus ! Blablablabla !", alors qu'il ne fait absolument pas froid chez moi! Donc on lui a mis des pulls, des chaussettes, mais alors c'était l'angoisse, je me disais : "Mais mon dieu, il va mourir, je ne $\mathrm{m}^{\prime}$ en occupe pas bien, il a froid, je ne suis même pas foutue de me rendre compte qu'il a froid !" [...] J'ai aussi mon médecin généraliste qui s'était scandalisé : j'étais malade, le petit devait avoir sept mois et au moment où il me prescrit les médicaments, je lui dis : "Tenez compte du fait que j'allaite". I/ me dit : "Ah bon ? Mais vous allaitez encore à sept mois ? Mais vous ne vous rendez pas compte, vous n'arriverez jamais à le sevrer ! Alors là c'est foutu, le gamin a pris l'habitude, voilà !" Ça alors, la grosse morale!»

Ceci est encore plus marqué dans les témoignages des parents concernant leur recours aux professionnels de la petite enfance. Personnel de crèche (éducateurs-trices, auxiliaires de puériculture, directeurs-trices et assistant-e-s maternel-le-s) incarnent, aux yeux des parents interrogés, des références en matière de prime éducation. Décrits comme socialement moins distants que les professionnels de santé, tout en restant plus «fiables » que la famille, les acteurs de la petite enfance sont sollicités en particulier sur des questions d'éveil, de socialisation, d'étapes de développement (propreté, langage...), de gestion des limites (punition, etc.). Là aussi, de part et d'autre, l'enjeu réside dans la définition des "bonnes » et des " mauvaises» pratiques dans ces domaines, avec ce que cela suppose comme effet sur l'assurance ou la culpabilité des jeunes parents.

- Lena, enseignante, 29 ans, en couple, deux enfants âgés de 3 ans et 8 mois : "À la crèche, de temps en temps, j'étais quand même blessée, quand c'est des trucs durs et que j'avais l'impression que ça pouvait venir de moi, que je pouvais influer sur quelque chose, ça me... Il y en avait une à la crèche, à l'époque où Maël tapait tout le monde, elle me le disait vraiment comme s'il était trop pénible et qu'elle attendait quelque chose de moi, [...] Alors moi : "Pardon je suis désolée !", mais zut, est-ce qu'elle attend que je le dispute en fait ? Ça me stressait ! [...] ça me faisait un poids incroyable ! »

Les professionnels de la santé et de la petite enfance, tout en relayant des principes propres à leur champ d'intervention, promeuvent ainsi des pratiques et des modèles plus ou moins explicites vis-à-vis desquels les parents sont jugés et/ou se jugent eux-mêmes. Ces modèles ne se réduisent pas à des normes médicales, sanitaires ou éducatives. Ils véhiculent aussi des normes sociales et morales, en premier lieu des normes genrées de parentalité à travers notamment ce que Anne-Sophie Vozari (2011) qualifie d'une "idéologie de la primauté maternelle » : "L'idée contemporaine d'absence de norme sociale de la vie familiale se fonde en fait sur des normes implicites de genre, pensées comme naturelles. Les idéologies de la dyade mère/enfant, véritables dogmes en matière de maternité, sont largement présentes et véhiculées [notamment] par les professionnelles de la petite enfance» (2011, p. 87). Les pères et les mères rencontrés rendent compte en effet de la prévalence de la norme de primauté maternelle entérinée dans les discours des professionnels de la santé comme de la petite enfance, et des effets différenciés que cela a sur eux. Dans le domaine de la santé, les avis et les prescriptions sont principalement adressés aux mères, ne laissant souvent aux pères qu'une place incertaine (Bigot et al., 2005), ce que déplorent certains d'entre eux qui racontent s'être sentis « mis à l'écart».

- Paul, cuisinier, en couple, 32 ans : " Je suis allé au cours prénatal... Enfin, la première fois, j'y étais. Après elles $m^{\prime}$ ont dit : "ça ne sert à rien que vous veniez, c'est pour la maman". Alors bon, je n'y suis plus allé, mais bon....

Dans le domaine de la petite enfance, de nombreux parents insistent également sur le poids des représentations

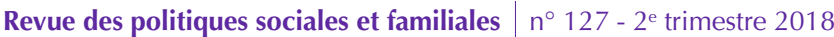

51 Parentalité 
de genre dans les conseils ou les avis qu'ils reçoivent. Là aussi, les mères sont considérées comme les interlocutrices privilégiées et comme responsables des aspects importants de la vie de l'enfant (Giampino, 2016). $C^{\prime}$ est donc aux mères que s'adressent plus volontiers les personnels de la petite enfance, aux mères également que $s^{\prime}$ adressent les prescriptions et parfois les reproches pour qui ne s'y conforme pas. Ce qu'explique notamment Julia (enseignante, en couple, 38 ans, mère de deux enfants âgés de 3 et 1 ans) :

- "Il y a tout le temps des petits commentaires sur la maman et le papa, c'est carrément des rôles différents $q u$ 'on est censés jouer. Récemment, ils ont dit à Laurent : "Il faut que maman coupe ses ongles". Donc il est rentré pour dire : "Il faut que MAMAN coupe les ongles !". II y a clairement : "C'est maman qui s'occupe des soins de l'enfant ou qui n'a pas fait son travail correctement !". Une fois, Laurent avait laissé des médicaments dans le casier, il avait appelé la crèche pour me faire dire de les ramener. À la crèche, ils m'ont dit : "Mais j'hallucine ! Maintenant $\mathrm{c}^{\prime}$ est les papas qui appellent pour dire aux mamans ce qu'il faut faire! C'est le monde à l'envers !". IIs rigolaient aussi un peu, mais c'était quand même complètement à l'opposé de comment ils conçoivent les choses...".

Fondées sur la promotion des « savoirs psy » (savoirs popularisés issus de la psychologie) et du lien mère-enfant, en particulier dans l'univers de la petite enfance (Neyrand, 1999), ces prescriptions normatives ont des effets genrés : d'abord parce qu'elles s'adressent essentiellement aux mères - les responsabilisant et déresponsabilisant les pères - et ensuite parce qu'elles valorisent comme critère de bonne parentalité des «qualités féminines ».

Cependant, si les parents témoignent être l'objet d'incitations à se conformer à des normes sanitaires et éducatives genrées relativement partagées par les différents professionnels, ils témoignent aussi faire face à des messages divergents, voire contradictoires, et de virulents débats concernant certaines pratiques, notamment l'allaitement.

- Marina, demandeuse d'emploi, en couple, 35 ans, un enfant âgé de 8 mois : « Vous avez des discours qui sont pratiquement contradictoires d'une équipe à l'autre, et ça c'est déstabilisant quand vous voulez vraiment bien faire et que vous ne savez pas trop et qu'on vous dit : "Mais là, faut faire ci ! Là, faut faire ça !", alors vous suivez la première sage-femme qui vous dit un truc et puis après vous changez d'équipe, et elle vous dit : "Ah non, mais non! Faut faire comme ça !". Moi je trouve que sur l'allaitement c'est vraiment compliqué !».

L'évolution des savoirs et les différences de statut, de formation et de logiques professionnelles entre les professionnels - notamment entre médecins et sages-femmes, entre éducatrices, puéricultrices et auxiliaires de puériculture (Geay, 2014) - semblent pouvoir expliquer en partie ces divergences. Toutefois, ces débats entre professionnels empruntent à d'autres registres que les registres professionnels ("savants », médicaux, psychoéducatifs). En matière de maternité, il n'est pas rare que les professionnels, de la santé comme de l'enfance, se réclament du " bon sens ", de la tradition (Gojard, 2000), de " la nature ou de l'instinct maternel » ou de leur propre expérience de parents (Giampino, 2016) pour promouvoir une approche plutôt qu'une autre, qu'il s'agisse d'allaitement, d'alimentation, de sommeil ou de prime éducation. Dès lors, $\mathrm{s}^{\prime}$ il existe une forte injonction des professionnels à adopter de « bonnes pratiques » ou de " bons comportements » de parents, les normes sanitaires et éducatives pour y parvenir sont parfois plurielles, équivoques, voire concurrentes (Chauffaut et Dauphin, 2012). Allaitement à la demande ou allaitement à heure fixe ; «faire comme on le sent » ou « faire comme il faut »; lâcher prise ou reprendre le contrôle ; n'être ni trop distant-e ni trop fusionnel-le; ni trop autoritaire ni trop permissif-ve : les discours des professionnels relatés par les parents se manifestent dans une tension constante entre des figures repoussoirs : celles du parent laxiste et du parent maltraitant, celles de l'enfant roi et de l'enfant délaissé (Serre, 1998). Dès lors, les messages relèvent parfois moins de la transmission $d^{\prime}$ indications sur ce qu'il convient de faire concrètement que de la promotion de modèles " en creux » dont le contenu reste implicite et flou. Du point de vue des parents, tout se passe bien souvent comme si on leur prescrivait un idéal du bon parent, et de la bonne mère en particulier, sans en définir de contenus unifiés :

- Julia : "En fait, il n'y a pas vraiment UN modèle de ce que c'est une bonne maman, donc c'est... au final, c'est chacun [c'est-à-dire chaque interlocuteur] qui impose ses visions de ce qu'on est censés faire ».

L'injonction à " être un bon parent » et à adopter les bonnes pratiques, si elle est exprimée par tous, revêt des contenus différents et plus ou moins explicites en fonction des prescripteurs, mais aussi en fonction du parent à qui elle s'adresse, de son genre et de son milieu d'origine supposé.

Revue des politiques sociales et familiales $n^{\circ} 127-2^{e}$ trimestre 2018

52 Parentalité 
Le recours à la famille et à l'entourage :

\section{entre soutien et critiques}

Les membres de la famille et l'entourage sont bien sûr des interlocuteurs et des recours de premier ordre à l'arrivée $d^{\prime}$ 'un enfant. Les familles, en particulier les grands-mères, sont le plus souvent sollicitées pour apporter du soutien et une aide domestique ou de garde d'enfant. À ce titre, le recours à la famille concerne des parents de tous les milieux, mais la solidarité familiale est plus opérante lorsque la famille réside à proximité. Toutefois, en matière $d^{\prime}$ informations, le recours à la famille est rapidement abandonné par de nombreux parents, pour deux raisons principales : les savoirs familiaux entrent en concurrence avec les prescriptions des professionnels et ils sont le plus souvent considérés par les parents rencontrés comme " n'étant plus d'actualité », et ce en particulier pour les parents les plus proches des professionnels. Au-delà de cet argument, on observe également que certains parents se montrent réticents à se conformer aux avis de leurs propres parents ou beaux-parents. C'est parfois même l'objet de conflits, notamment entre mères et belles-mères et filles, ces dernières dénonçant notamment les commentaires intrusifs ou les jugements critiques dont elles se sentent l'objet. Si la famille reste une importante pourvoyeuse d'informations et de prescriptions concernant la transmission des valeurs familiales, en particulier pour les familles les plus modestes, elle est bien souvent présentée également comme une source de critiques concernant les pratiques et les choix parentaux des enquêtés.

Ces ambivalences sont également observées en ce qui concerne le recours à des amis ou des collègues. Le fait d'avoir des amies proches, elles-mêmes mères, est un facteur de soutien très important pour certaines mères, en particulier pour celles qui sont éloignées de leur famille, et ce tous milieux confondus. Toutefois, certaines mentionnent là encore leurs réticences à « recevoir » ce qu'elles estiment être des jugements de la part des autres mères. Elles témoignent par exemple de phénomènes de "compétition ", tout du moins de comparaison entre parents. Sur fond de jugements réciproques, il s'agirait parfois de comparer les talents des enfants, mais aussi les « compétences » voire les "performances " parentales. Des compétitions en particulier vouées au culte de " la mère parfaite ».

- Clémentine, coordinatrice dans une association, en couple, 35 ans : "Il y a un nouveau groupe sur Facebook qui s'appelle les "Milk», c'est un acronyme pour "Mothers I'd like to kill" [" les mères que j'aimerais tuer »]. Et c'est génial, c'est un site où publient toutes les femmes comme moi qui n'arrivent pas du tout à tout gérer et qui partagent sur les trucs de ces mères parfaites qui ont l'air d'avoir une maison qui est toujours nickel, qui sont trop belles, trop bien habillées, trop calmes! [...] Alors que toi, tu n'en peux plus !».

On le voit, l'arrivée d'un premier enfant se caractérise, dans les récits de certains parents, comme une période de socialisation à de nouvelles normes médicales, sanitaires, éducatives ou familiales. Mais c'est aussi et surtout une période de socialisation à un nouveau rôle qui soumet les jeunes parents aux avis, aux regards, aux jugements voire aux injonctions d'une pluralité d'interlocuteurs et prescripteurs des « bonnes » manières d'être parents. Au-delà du contenu des normes qui sont adressées, les entretiens montrent que le processus de normalisation lui-même - et donc la manière dont on s'adresse à eux - semble déterminant dans la manière dont les parents expérimentent les premiers temps de leur parentalité et gèrent leurs relations aux interlocuteurs, adoptant différentes stratégies de recours et/ou d'évitement.

\section{Des stratégies}

\section{de recours aux tactiques d'évitement}

Si la grande majorité des parents recourt, à un moment ou un autre, à tous ces interlocuteurs, les entretiens montrent des différences dans les tendances des parents à recourir à tel ou tel autre interlocuteur. Ces dernières semblent liées à la fois à leur type d'attentes (recherche d'information, de soutien moral ou domestique, de sociabilité...), à leurs profils socioculturels, mais aussi à leur manière de gérer le poids des attentes et prescriptions normatives auxquels ils se confrontent.

\section{Des tendances sociales}

\section{dans le choix des interlocuteurs}

Des tendances sociales se dessinent dans la description que les parents interrogés font de leurs démarches pour trouver de l'information, du soutien ou de l'aide. Si ce n'est pas systématique, on constate, à la suite notamment des travaux de S. Gojard (2010), que les parents de milieux relativement aisés et diplômés sollicitent plus volontiers les professionnels de santé, en particulier médecins, gynécologues et pédiatres. En recherche d'informations fiables et "savantes ", ils sont également plus souvent amenés à multiplier les recours auprès de différents professionnels, à diversifier leurs interlocuteurs, à mener leurs propres recherches documentaires, tout en prenant plus volontiers leur distance avec les savoirs familiaux. Les parents de milieux plus modestes semblent avoir tendance à privilégier d'abord l'accessibilité des informations et la proximité des 
interlocuteurs. À ce titre, les professionnels de la petite enfance sont souvent perçus comme plus accessibles plus présents au quotidien - que ceux de la santé ; ces derniers paraissent à la fois éloignés des parents les plus isolés socialement et les plus éloignés des zones urbaines.

Cependant, lorsque les parents sont davantage à la recherche de sociabilité, de soutien, de réassurance ou de réconfort, les contrastes sociaux semblent beaucoup moins saillants, là où les dimensions de genre sont déterminantes. Ce sont les mères, quasi exclusivement, qui disent rechercher des formes de soutien, en particulier au sortir de la maternité, aux cours des congés maternité et à plus forte raison, parentaux. L'isolement des mères - qu'il concerne des profils aisés ou des profils plus modestes, des mères célibataires ou des mères en couple - suscite le même type d'attentes et de besoins : être moins seule face aux questions, aux doutes, à la responsabilité, et se sentir rassurée sur le fait que ce que l'on vit peut être considéré comme « normal ». À ce titre, elles privilégient le recours à la famille, à l'entourage et, pour certaines, à des groupes de pairs : associations, lieux d'accueil enfants-parents, réseaux sociaux sont autant de ressources mobilisées par des femmes ayant avant tout en commun de traverser des périodes de solitude.

Enfin, certains parents, à la fois éloignés de leur famille, des professionnels et des sources d'information témoignent de très faibles niveaux de connaissance des recours possibles et des aides qu'ils pourraient mobiliser. C'est le cas d'Aïcha (demandeuse d'emploi, célibataire, 44 ans) qui, enceinte de sept mois, et dans une situation de grande vulnérabilité, ne sera suivie médicalement et socialement qu'une fois identifiée à l'hôpital, à la suite d'une urgence médicale. De même, Françoise (demandeuse d'emploi, en couple, 50 ans), durant sa grossesse tardive, ignore les différentes possibilités de suivi et les sources d'informations possibles en dehors de ses rendez-vous gynécologiques et n'aura aucun autre interlocuteur. Cet isolement, cet éloignement des interlocuteurs, peut aussi être le lot de parents plus informés par ailleurs, mais résidant en zones rurales ou traversant des situations critiques. Ainsi, Adeline (agricultrice, en couple, 41 ans) et Emma (mère au foyer, en couple, 36 ans), pourtant relativement bien informées, se trouveront malgré tout très isolées pendant la grossesse ainsi qu'à la suite de l'accouchement. Alitées, résidant en zone rurale, leurs uniques interlocuteurs durant de longues périodes seront les aides et sages-femmes à domicile. Pour certains parents, les interlocuteurs sont peu accessibles et les choix de recours sont donc limités.
Les jeunes parents ont donc différentes pratiques d'information et de recours à différents interlocuteurs en fonction de leurs profils, de leurs attentes et de leurs possibilités. Toutefois, les entretiens montrent qu'au-delà des tendances évoquées jusqu'ici les stratégies de recours et de non-recours des parents s'expliquent aussi à travers leurs différentes manières de recevoir les prescriptions normatives du « bon parent » et de se conformer, de résister ou d'échapper au poids des modèles de parentalité véhiculés par ces interlocuteurs.

\section{La mise à distance d'un idéal de parentalité}

Dans les premiers temps de l'arrivée d'un premier enfant, la plupart des parents rencontrés se conforment aux prescriptions des professionnels, en particulier aux prescriptions des professionnels de santé. Toutefois, les entretiens montrent que, rapidement, nombre de parents adoptent des stratégies ou des tactiques visant à gérer différemment, voire à éviter l'afflux et le poids des prescriptions. Ceci est notamment le cas de parents exigeants qui sont dans une quête des bonnes pratiques et d'un idéal de parentalité, et qui multiplient les interlocuteurs et les sources d'informations; des parents qui peuvent avoir tendance à se conformer d'autant plus aux prescriptions qu'ils font de la parentalité un critère de réussite et d'accomplissement personnel (Dauphin, 2014 ; Martin, 2003 ; Neyrand, 1999). Or, au bout de quelques mois, ces parents " exigeants", voire " perfectionnistes ", plutôt présents dans les milieux aisés et diplômés, racontent bien souvent " avoir mis la barre très haut, peut-être trop... », " avoir voulu trop bien faire ", " avoir trop écouté » et avoir progressivement pris conscience de la nécessité de prendre des distances vis-à-vis d'un idéal de parentalité trop élevé et de ses prescripteurs.

Ainsi, Léna a pris un long congé parental pour s'occuper de ses enfants. Elle se documente en permanence, est avide de lectures, multiplie les interlocuteurs et investit une grande partie de son temps à développer ce qu'elle qualifie elle-même « d'expertise » de parents : « Il y a un idéal, il y a des idéaux que tu as à la base, et en fait ça complique la tâche. Selon où tu t'es mis la barre, et moi je pense que je m'étais mis la barre hyper haute tout de suite, en me disant: "Les enfants il faut les élever bien, il faut leur faire à manger moi-même, bio, il faut les allaiter longtemps, etc." Enfin, tout ça, c'était mes principes avant... Après, quand tu reviens dessus, c'est dur parce que c'est un sentiment d'échec ». Lena raconte avoir progressivement évité de partager certaines préoccupations avec sa mère et le personnel de la crèche et avoir privilégié

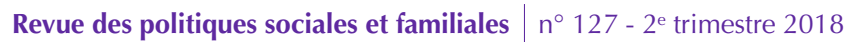


les recherches personnelles et les « copines mamans» rencontrées dans un lieu d'accueil enfants-parents (Laep).

Les parents rencontrés témoignent d'une prise de conscience plus ou moins progressive du poids des normes qui pèsent sur leur entrée en parentalité et qui sont relayées par de multiples interlocuteurs. Laurent (ingénieur, en couple, 41 ans), dit constater lui aussi une augmentation des exigences vis-à-vis des parents qui accompagne le « centrage sur l'enfant » (Bachmann et al., 2016) :

- "La société a un poids aujourd'hui qui est beaucoup plus fort [...] C'est sûr qu'aujourd'hui, on te pousse beaucoup à utiliser le dernier équipement aux normes, le dernier biberon qui a moins de pesticides, le bio, t'as une grosse pression là-dessus de la part de l'entourage, des amis aussi effectivement. Et tu le fais ! Tu le fais ! [Rires] Mais c'est une pression, elle est double en fait : les nouvelles générations qui, pour certaines, te disent que tu n'en fais pas assez et les vieilles générations qui te disent que t’en fais trop!»

Face au sentiment de n'être pas suffisamment à la hauteur d'un idéal trop élevé ou insaisissable, d'être déstabilisés par la multiplication des messages, mais aussi face à des théories contradictoires les rendant progressivement sceptiques et incrédules, nombre de parents adoptent des stratégies de mise à distance de l'idéal, et avec lui des prescripteurs de normes qui contribuent à le façonner ou l'imposer.

\section{L'évitement, la résistance et la recherche d'alternatives}

Ainsi, un autre grand motif de non-recours émerge des témoignages: I'incertitude, l'angoisse, les reproches, les jugements auxquels les parents cherchent à échapper. De fait, des injonctions trop nombreuses ou trop pesantes provoquent des formes de repli, de retrait. Face aux conseils parfois contradictoires et à la concurrence des normes, les parents rendent compte, lors des entretiens, de stratégies visant à éviter les doutes et les dilemmes : par exemple, "s'en remettre à soi-même », opérer une stricte sélection des interlocuteurs ou décider de n'avoir qu'un seul interlocuteur pour toutes les questions.

- Clémentine, 35 ans, en couple, coordinatrice dans une association : "Une des choses que j'ai réalisée dès la grossesse, c'est que tout le monde a un avis sur tout. Et quelqu'un m'a dit un jour : "Choisis une ou deux référentes maximum, ça peut être ta mère, ta grand-mère, qui tu veux. Mais choisis une seule personne et laisse dire les autres. Tu te focalises". C'est ce que j'ai fait, c'est ma sage-femme, je n'écoute qu'elle».

Pour certains parents, ce n'est pas tant la concurrence des interlocuteurs qu'il s'agit de fuir que la pression normative elle-même. À ce titre, l'évitement de la pression ou des reproches peut tout aussi bien concerner la famille que les professionnels et prendre différentes formes. Dorian (éducateur, 32 ans) et Mélanie (graphiste, 29 ans), en couple, cherchent ainsi à échapper aux angoisses de la grossesse comme à la pression de l'encadrement médical en combinant suivi de grossesse minimaliste et désobéissance aux consignes de la maternité :

- Mélanie : "J'ai juste vu un gynécologue pendant ma grossesse. Sinon, non, je n'ai pas été suivie plus que ça. J'ai fait un seul cours de préparation à l'accouchement mais je n'avais pas envie... Moi, je n'étais pas angoissée, je $n^{\prime}$ avais pas envie qu'on me refile des angoisses que je n'avais pas, des questions que je me posais pas... Du coup, je n'ai pas continué ! [Rires] [...] On nous donne des consignes à la maternité. Mais bon, tout ça, nous, on l'a fait vraiment au feeling [...] On n'a pas écouté, ni rien ni personne, on s'est écoutés nous ».

Certains parents mettent ainsi en œuvre des stratégies de résistance aux prescriptions des professionnels, qui se manifestent notamment par des "désobéissances ". Celles-ci se traduisent le plus souvent sous des formes discrètes, des "faux-semblants » mais aussi parfois par des réactions plus frontales. Céline (doctorante, en couple, 37 ans) refuse, par exemple, de se soumettre à ce qu'elle estime être une question à la fois intrusive et empreinte de jugement de la part de la gynécologue : "Tout le monde te dit ce que tu dois faire! Une des choses sur lesquelles j'ai eu beaucoup d'avis, c'est sur l'allaitement! Par exemple, le rendez-vous avec la gynéco au septième mois, la question a été tournée de cette façon-là (et je me suis dit "faut que je la note" !), c'était : "Et bien sûr vous allez allaiter?" La question a été posée comme ça : "Et bien sûr vous allez allaiter ?". Bon, après, c'est mon caractère aussi, j'ai répondu : "Et bien sûr que non !", alors qu'en vrai, pour moi la question reste ouverte [...]. Pour moi, c'est une question qui n'était pas facile à trancher, mais du coup j'ai décidé que, quand on me pose la question, de répondre: "ça ne vous regarde pas. C'est une question privée" ». 
À un autre niveau, certains parents s'engagent dans une recherche d'alternatives aux recours classiques considérés précisément comme trop normatifs ou trop intrusifs. Mélanie et Dorian, à l'instar de beaucoup d'autres, militent pour un retour au " naturel » et à " l'instinct » pour contrer ce qu'ils estiment être un surencadrement et une surmédicalisation de la grossesse et de la naissance. De même, Anita (demandeuse d'emploi, 27 ans) fera le choix de l'accouchement à la maison et d'un recours exclusif à des sages-femmes, qu'elle considère comme étant davantage à l'écoute que les gynécologues. Cette tendance s'observe aussi vis-à-vis du domaine de la petite enfance. La mise à distance des prescriptions normatives sous l'argument de "s'écouter soi-même » ou de «faire comme on le sent " est ainsi largement partagée au sein de différents milieux sociaux. A l'image des des classes populaires, comme le souligne S. Gojard (2000, p. 504) : "les membres des classes supérieures se réclamant de puériculture à tendance psy tiennent exactement ce type de discours selon lequel l'expérience maternelle (quand ce n'est pas l'instinct) prime sur les prescriptions savantes, fondées sur un savoir abstrait et qui risquent toujours de n'être pas adaptées au cas singulier qui est le leur ».

De la même manière, le partage d'expériences entre les parents, et principalement entre les mères, est souvent présenté comme une alternative aux recours traditionnels. Rares sont les mères qui excluent totalement de faire appel à des professionnels pour les aider en matière de périnatalité ou de prime éducation, mais certaines leur préfèrent l'échange entre pairs.

- Anita : "On se sent très seule, si le papa travaille... On est toute seule à la maison... Donc là, pour moi, l'idée c'est surtout de rencontrer d'autres mamans, pour moi, c'est la meilleure chose [...]. Parce que les organismes [elle parle de la PMI] ils vont te donner la théorie : "Il faut faire comme ça, comme ci". Alors que si tu discutes avec d'autres mamans, c'est là où tu vas voir que l'une, elle fait comme ça, l'autre, elle fait comme ci, chacune fait à sa manière et, finalement, il y des choses qui marchent».

Qu'il s'agisse de "groupes de mamans » qui se créent sur les réseaux sociaux, dans les cours de préparation à la naissance ou de yoga prénatal, qui se rencontrent dans des Laep, des ateliers de loisirs, des associations, et même des squares, tous ont en commun de faire du partage d'expériences un recours de soutien à la parentalité. La création de liens avec d'autres mères est décrite comme permettant d'accéder à des informations, de partager des expériences et moments de sociabilité mais aussi de se protéger du regard subjectif de la famille et du regard potentiellement surplombant des professionnels.

\section{Les stratégies de protection :}

\section{être mère sous le regard des autres}

S'il s'agit d'éviter les injonctions contradictoires, la pression ou le surencadrement, c'est bien souvent aussi à la peur et la culpabilité d'être un "mauvais parent » qu'il s'agit d'échapper. Nombre de parents, et de mères en particulier, témoignent de leurs tentatives de fuir prescriptions, conseils, avis non sollicités et qui sont vécus comme des critiques. À ce titre, tous les interlocuteurs sont susceptibles d'être perçus comme jugeants et culpabilisants par les parents, qu'il s'agisse de l'entourage, des professionnels, des pairs, comme des auteurs de livres spécialisés, etc.

- Magali, institutrice, célibataire, 35 ans : «On se sent jugé parfois. Particulièrement par la nounou et même par mes parents, même si ce n'est pas dit comme ça, même si c'est toujours justement sous forme de conseils. Quand on donne un conseil, c'est que quelque chose est mal fait finalement, donc quelque part, on le prend comme ça... comme un reproche, oui. Oui, c'est ça être parent aussi ».

Là où les prescriptions sont avant tout adressées aux mères, on trouve l'expression chez les femmes de forts sentiments de culpabilité liés à la crainte d'être jugée en tant que mère :

- Marine, animatrice musicale, 30 ans : "Je me dis clairement "je ne suis pas une professionnelle de la question et si on a de bons conseils à me donner, je suis preneuse !" Mais, en même temps... je suis sensible à la façon dont on me représente comme mère [...]. Je trouve que c'est hyper difficile de savoir comment gérer [...] mais tu ne peux pas réceptionner tous les conseils et tu n'as pas envie qu'on te prenne de haut non plus... qu'on te culpabilise ».

Bien que les choses évoluent, les enfants restent en grande partie une " affaire de femmes ", et les conceptions familialistes fondées sur la division traditionnelle des rôles de genre perpétuent des tendances à la surresponsabilisation, voire à la culpabilisation des mères (Knibiehler et Neyrand, 2004 ; Blöss, 2016) ; des tendances qui se 
traduisent dans les remarques des professionnels, les injonctions des institutions, les attentes de la société et qui ont des effets différenciés, comme l'expliquent Laurent et Julia lors de deux entretiens individuels :

- Laurent : «Julia, elle-même, se met plus de pression ! Moi j'accepte beaucoup plus, j'accepte toutes les remarques et si je considère qu'elles sont nulles, je les ignore, mais Julia non [...] Par exemple, les remarques de la crèche. C'est pareil, moi je n'y fais pas attention. Moi, les remarques que je trouve non pertinentes, j'oublie tout de suite! Je ne me sens pas du tout attaqué... [...] Clairement, c'est les femmes qui se prennent plus... enfin, je ne sais pas, mais est-ce $q u$ 'elles sont plus pointilleuses ou est-ce qu'elles se prennent plus de remarques...».

- Julia : "Par rapport aux injonctions qu'on a de la crèche, Laurent, il dit : "On s'en fiche !". Bon, mais lui, il n'a jamais de commentaires de l'extérieur sur la manière dont il élève les enfants, il ne se sent pas responsable de ça. Tout ce qu'il peut faire c'est du "bénéfice", c'est au-delà des attentes de la société, donc c'est toujours bien ce qu'il fait. Alors que les femmes, on est critiquées sur la manière dont on fait n'importe quelle chose. Tout problème d'éducation, c'est censé être la faute de la maman ».

Ces attentes sociales différenciées ont donc des effets sociaux : outre le fait qu'elles sont susceptibles de mettre à l'écart et/ou de déresponsabiliser les pères en matière de soin et d'éducation auprès des enfants petits ${ }^{(3)}$, elles peuvent contribuer à la fragilisation de mères fréquemment mises en cause, et confrontées à la peur d'être de « mauvaises mères "(Cardi, 2007). Les normes de parentalité, telles qu'elles sont prescrites, perpétuent encore bien souvent un modèle dans lequel être mère suppose de fournir plus de preuves pour démontrer sa qualité et échapper aux soupçons.

- Enquêteur : Vous avez à peu près les mêmes préoccupations, votre conjoint et vous?

- Céline : «Oui, oui. Mais, par contre, mon conjoint a beaucoup moins de culpabilité que moi ! Et ça c'est vraiment, vraiment lié, c'est tellement une évidence! au fait que tout ce qu'il fait est valorisé ! Et que moi, ce que je fais, ça n'est jamais assez... ».
Dans ce contexte, certaines mères adoptent des stratégies pour être de moins en moins confrontées à des conseils, des avis ou des injonctions qui pourraient les déstabiliser. Ceci constitue aussi l'une des raisons pour lesquelles des mères privilégieront le recours à des groupes de pairs plutôt qu'à des professionnels. D'autres se montrent progressivement de plus en plus réticentes aux conseils de tiers et adoptent de véritables stratégies défensives et de protection. $C^{\prime}$ est le cas de Julia qui, à la suite des retours et des remarques des professionnelles de la crèche accueillant sa fille, décide d'éviter de s'exposer aux jugements : "Désormais, je ne montre plus que je ne sais pas quoi faire. Je m'étais résolue à ne plus montrer de faiblesses en tant que maman. [...] C'était un peu la solution que j'avais trouvée : ne plus poser de questions, ne plus montrer qu'on a des doutes [...] Finalement, on a l'impression de recevoir en permanence des critiques : "faut pas faire comme ci, comme ça !" Et même si ce n'est pas des critiques réelles basées sur des choses que tu as vraiment faites, ce sont des injonctions sur ce qu'on est censé faire... Mais on a rarement des moments où on nous félicite pour ce qu'on fait ! ».

D'autres, enfin, sont clairement sur la défensive, et cherchent à se protéger des regards désapprobateurs, voire de tous les regards.

- Emma : "J'avais une grande difficulté ne serait-ce que d'aller dans les services publics [...]. Bon, mais c'est toujours une difficulté que j'ai, sur ce que les gens vont bien pouvoir penser de moi si mon bébé se roule par terre et fait un caprice, ça c'est terrifiant! Terrifiant! Et je lui donne des gâteaux, comme ça elle ne le fait pas ! [Rires] [...] Mais ça, quand j'étais vraiment une toute jeune maman, comprendre pourquoi elle pleurait et ce que les gens allaient bien pouvoir penser de ma réaction, c'était... voilà ! Ouf, ça m'a empêché de sortir ! Ah oui, ça m'a empêché de sortir!»

\section{Conclusion}

L'analyse des récits des parents sur leur « entrée en parentalité » permet de saisir ce qui caractérise la socialisation des jeunes parents à des normes de parentalité, à travers leurs rapports aux différents interlocuteurs auxquels ils font face et/ou auxquels ils recourent. Les discours des professionnels de la santé, de la petite enfance, de la famille et de l'entourage contribuent à la fabrique de

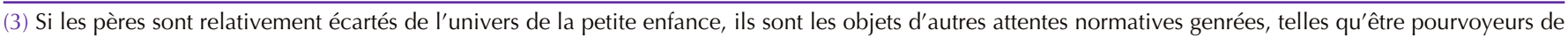
ressources ou assurer un rôle d'autorité auprès des enfants plus grands, notamment.

Revue des politiques sociales et familiales $\mid n^{\circ} 127-2^{\text {e }}$ trimestre 2018 
différents modèles de parentalité : des modèles savants ou traditionnels, de pères et de mères, qui véhiculent différentes normes du « bon parent ».

Au-delà du contenu de ces normes, les manières dont elles sont adressées et reçues par les parents semblent tout aussi importantes pour comprendre comment ils expérimentent les premiers temps de leur parentalité, se conforment ou résistent aux prescriptions qu'ils reçoivent et recourent ou non à des interlocuteurs et des aides extérieures. Si la recherche $d^{\prime}$ informations ou de soutien des parents auprès de ces différents interlocuteurs dépend beaucoup de leurs attentes, et peut varier selon les profils socioprofessionnels, notamment, on constate qu'elle dépend aussi de la manière de percevoir et de gérer ces prescriptions. La parentalité se raconte aussi à travers des manières de réagir à de fortes attentes sociales qui balisent le parcours de parents sommés de questionner leurs propres pratiques au regard des « bonnes » normes. Qu'ils y adhèrent ou non, les jeunes parents ne peuvent que difficilement échapper à I'injonction qui leur est faite d'être de « bons parents ». Or, il s'agit là d'une injonction souvent perçue comme d'autant plus paradoxale que les normes et les pratiques prescrites ne sont pas unifiées et varient selon les interlocuteurs et selon le profil des parents à qui elles sont adressées.

Dès lors, les parents mettent en œuvre diverses stratégies vis-à-vis des prescripteurs, qu'ils soient professionnels ou familiaux ; les postures des parents rencontrés témoignent de leurs différentes manières de réceptionner ces prescriptions, pour en adopter certaines, les éviter, les relativiser ou les refuser. Là encore, les pratiques et les stratégies dépendent des profils des parents (profil socioprofessionnel, situations sociale et résidentielle, genre), de leur niveau de connaissance et d'assurance (I'arrivée du premier enfant est bien spécifique dans ce domaine) mais aussi du degré de "normalisation » auquel ils sont ou ont été soumis. À ce titre, le spectre de «la mauvaise mère » est l'une des figures normatives les plus pesantes sur le quotidien parental des femmes contribuant à fragiliser certaines et/ou à les éloigner des institutions, des professionnels et de potentiels soutiens à la parentalité. Des constats qui sont susceptibles d'alimenter et de diversifier la compréhension des phénomènes de non-recours, notamment dans le domaine de l'accompagnement à la parentalité.

- Bachmann L., Gaberel P.-E., Modak M., 2016, Parentalité : perspectives critiques, Lausanne, les éditions de l'École d'études sociales et pédagogiques.

- Bigot S., Céroux B., De Ridder G., 2005, Le suivi médical de la grossesse. Les pères en devenir face à leur implication, Didier Le Gall D. (dir.), Genres de vie et intimités. Chroniques d'une autre France, Paris, L'Harmattan, p. 135-151.

- Blöss T., 2016, Devoirs maternels. Reproduction sociale et politique des inégalités sexuées, Actes de la recherche en sciences sociales, $n^{\circ} 214$, p. 46-65.

- Boucher M. (dir.), 2011, Gouverner les familles. Les classes populaires à l'épreuve de la parentalité, Paris, L'Harmattan.

- Boyer D., Céroux B., 2010, Les limites des politiques publiques de soutien à la paternité, Travail, genre et sociétés, $\mathrm{n}^{\circ} 24$, p. 47-62.

- Cardi C., 2007, La mauvaise mère : figure féminine du danger, Mouvements, $\mathrm{n}^{\circ}$ 49, p. 27-37.

- Chauffaut D., Dauphin S., 2012, Normes de parentalités : production et réception. Revue de littérature, Politiques sociales et familiales, $\mathrm{n}^{\circ} 108, \mathrm{p} .108-115$.

- Chauvière M., 2008, La parentalité comme catégorie de l'action publique, Informations sociales, $\mathrm{n}^{\circ} 149$, p. 16-29.

- Dauphin S. 2014, Parentalité et politique de la famille, Politiques sociales et familiales, $\mathrm{n}^{\circ} 118$, p. 59-64.

- Geay B., 2014, Les relations entre parents et personnels d'accueil de jeunes enfants. La transmission des normes au prisme des rapports entre classes sociales, Politiques sociales et familiales, $\mathrm{n}^{\circ} 118$, p. 35-44. 
- Giampino S., 2016, Développement du jeune enfant. Modes d'accueil, Formation des professionnels, rapport remis au ministre des Familles, de l'Enfance et des Droits des femmes, 9 mai 2016. Site internet consulté le 27 août 2018 : https://www.egalite-femmes-hommes.gouv.fr/wp-content/uploads/2016/05/RapportGiampino-vf.pdf.

- Gojard S., 2010, Le métier de mère, Paris, La Dispute, collection Corps, santé, société.

- Gojard S., 2000, L'alimentation dans la prime enfance, diffusion et réception des normes de puériculture, Revue française de sociologie, $\mathrm{n}^{\circ} 41$, p. 475-512.

- Knibiehler Y., Neyrand G. (dir.), 2004, Maternité et parentalité, Rennes, École nationale de santé publique.

- Manier M., 2016, Jeunes parents dans la tourmente ? Vécu, attentes et recours des parents à l'arrivée d'un premier enfant, rapport pour le compte de la Caf des Alpes-Maritimes, Université Nice Sophia Antipolis (Urmis).

- Martin C., 2014, "Être un bon parent » : une injonction contemporaine, Rennes, Presses de l'École des hautes études en santé publique, collection Lien social et politiques.

- Martin C., 2003, La parentalité en question. Perspectives sociologiques, rapport pour le Haut Conseil de la population et de la famille, Paris, La Documentation, collection Rapports publics. Site internet consulté le 27 août 2018 : http://www.ladocumentationfrancaise.fr/rapports-publics/034000552/index.shtml.

- Menuel J., 2012, Devenir enceinte. Socialisation et normalisation pendant la grossesse : processus, réceptions, effets, Dossier d'étude, Caisse nationale des Allocations familiales, nº 148.

- Moeneclaey J., Crépin A., 2016, Les attentes et besoins des parents en matière d'accompagnement à la parentalité, l'e-ssentiel, n 165.

- Neyrand G., 2011, Soutenir et contrôler les parents. Le dispositif de parentalité, Toulouse, Érès.

- Neyrand G. 1999, Savoirs et normes sociales en matière de petite enfance, Recherches et Prévisions, $\mathrm{n}^{\circ}$ 57-58, p. 3-15.

- Oria N., Camus J., 2012, Avoir un premier enfant : un rite d’institution, Recherches familiales, n 9, p. 49-59.

- Pailhé A., Solaz A., 2006, Vie professionnelle et naissance : la charge de la conciliation repose essentiellement sur les femmes, Populations \& Sociétés, n 426.

- Pioli D., 2006, Le soutien à la parentalité : entre émancipation et contrôle, Sociétés et jeunesse en difficulté, [en ligne], n¹ | Printemps 2006, mis en ligne le 23 octobre 2006, consulté le 27 août 2018. URL : http://journals. openedition.org/sejed/106.

- Régnier-Loilier A., 2009, L'arrivée d'un enfant modifie-t-elle la répartition des tâches domestiques au sein du couple?, Population \& Sociétés, $\mathrm{n}^{\circ} 461$.

- Serre D., 1998, Le bébé « superbe » : la construction de la déviance corporelle par les professionnel(le)s de la petite enfance, Sociétés contemporaines, $\mathrm{n}^{\circ} 31$, p.107-127.

- Singly F. (de), 2009, Comment aider l'enfant à devenir lui-même ? Guide de voyage à l'intention du parent, Paris, Armand Colin.

- Vozari A.-S., 2011, Les ambivalences de I'intervention sociale en direction des familles. La protection maternelle et infantile entre "protection de l'enfance » et "Soutien à la parentalité ", sous la direction de Marc Bessin, Dossiers d'études, Caisse nationale des Allocations familiales, $n^{\circ} 136$. 